\title{
BOUNDING THE ORDERS OF FINITE SUBGROUPS
}

\author{
IAN J. LeARY AND Brita E. A. Nucinkis
}

Abstract

We give homological conditions on groups such that whenever the conditions hold for a group $G$, there is a bound on the orders of finite subgroups of $G$. This extends a result of P. H. Kropholler. We also suggest a weaker condition under which the same conclusion might hold.

\section{Introduction}

Let $R$ be a non-trivial unital ring. An $R$-module $M$ is said to be of type $F P_{n}$ if there is a projective resolution

$$
\cdots \rightarrow P_{n+1} \rightarrow P_{n} \rightarrow \cdots \rightarrow P_{0} \rightarrow M \rightarrow 0
$$

of $M$ over $R$ in which $P_{0}, \ldots, P_{n}$ are finitely generated. $M$ is said to be of type $F P_{\infty}$ if $M$ is $F P_{n}$ for each $n$. Similarly, $M$ is said to be of type $F P$ (resp. $F L$ ) over $R$ if there is a resolution of $M$ of finite length in which each term is a finitely generated projective (resp. free) module. For any discrete group $G$ and commutative ring $R$, the augmentation homomorphism $R G \rightarrow R$ gives $R$ the structure of a module for the group algebra $R G$. The group $G$ is said to be $F P_{n}$ (resp. $F P_{\infty}, F P, F L$ ) over $R$ if the $R G$-module $R$ is $F P_{n}$ (resp. $F P_{\infty}, F P, F L$ ) in the above sense. The cohomological dimension of $G$ over $R$, denoted by $\operatorname{cd}_{R}(G)$, is the projective dimension of $R$ as an $R G$-module. For further information concerning these definitions, see [2] or Chapter VIII of [3]. As usual, let $\mathbb{Q}$ and $\mathbb{Z}$ denote the rational numbers and the integers respectively. We prove the following.

Proposition 1. Let $G$ be a group with $\operatorname{cd}_{\mathbb{Q}}(G)=n<\infty$ and suppose that $G$ is of type $F P_{n}$ over $\mathbb{Z}$. Then there is a bound on the orders of finite subgroups of $G$.

2000 Mathematics Subject Classification. Primary: 20J05; Secondary: 19J05.

Key words. Finiteness conditions, finite subgroups.

The first author acknowledges support from EPSRC via grant GR/R07813, SFB 478 in Münster, the FIM at the ETH Zürich and the CRM Barcelona. 
A similar result was proved by P. H. Kropholler in Section 5 of $[6]$, under the extra hypothesis that $G$ should be $F P_{\infty}$ over $\mathbb{Z}$. His proof made use of the complete cohomology introduced by D. Benson, J. Carlson, G. Mislin and F. Vogel [1], [9] as will ours. (Complete cohomology can be viewed as a generalization of Tate cohomology.)

The conclusion does not hold for all groups of type $F P_{n-1}$ over $\mathbb{Z}$. K. S. Brown has shown [4] that for each $n>0$, the Houghton groups [5] afford an example of a group $G=G(n)$ such that:

(a) $G$ contains the infinite, finitary symmetric group;

(b) $\operatorname{cd}_{\mathbb{Q}}(G)=n$;

(c) $G$ is $F P_{n-1}$ over $\mathbb{Z}$.

The authors have recently constructed groups $G$ of type $F P_{\infty}$ over $\mathbb{Z}$ with $\operatorname{cd}_{\mathbb{Q}} G$ finite that contain infinitely many conjugacy classes of finite subgroups [7], and it was these examples that led to the authors' interest in Proposition 1. It is not known whether there is a bound on the orders of finite subgroups for every $G$ of type $F P$ over $\mathbb{Q}$. Some remarks concerning this question will be made at the end of the paper.

\section{Proofs}

Before starting, we recall a basic property of $F P_{n}$-modules. Suppose that $M$ is an $R$-module of type $F P_{n}$, and that

$$
P_{n-1} \rightarrow P_{n-2} \rightarrow \cdots \rightarrow P_{1} \rightarrow P_{0} \rightarrow M \rightarrow 0
$$

is a partial projective resolution of $M$ in which each $P_{i}$ is finitely generated. Then $K_{n-1}$, defined as the kernel of the map from $P_{n-1}$ to $P_{n-2}$, is finitely generated.

We shall also give a brief outline of Benson and Carlson's version of generalized Tate cohomology for arbitrary rings $R[\mathbf{1}]$. For $R$-modules $M$ and $N$ let $P \operatorname{Hom}_{R}(M, N)$ be the group of all $R$-module homomorphisms which factor through a projective, and let

$$
[M, N]=\operatorname{Hom}_{R}(M, N) / P \operatorname{Hom}_{R}(M, N) .
$$

For arbitrary $R$-modules $M$ let $F M$ be the free module on the set $M$ and $\Omega M$ is the kernel of the canonical projection $F M \rightarrow M$. Let $\Omega^{i} M=$ $\Omega\left(\Omega^{i-1} M\right)$. Then there is a well defined sequence of maps

$$
[M, N] \rightarrow[\Omega M, \Omega N] \rightarrow\left[\Omega^{2} M, \Omega^{2} N\right] \rightarrow \cdots
$$

and it is now possible to define the Tate cohomology group in degree zero as a direct limit as follows: 


\section{Definition.}

$$
\widehat{\operatorname{Ext}}_{R}^{0}(M, N)=\underline{\lim }\left[\Omega^{i} M, \Omega^{i} N\right] .
$$

From now on we shall concentrate on projective resolutions $P_{*} \rightarrow \mathbb{Z}$ of the trivial module $\mathbb{Z}$ over the group-ring $\mathbb{Z} G$. Let $K_{i}$ be the kernel of the map $P_{i} \rightarrow P_{i-1}$ for $i \geq 1$ and $K_{0}=\operatorname{ker}\left(P_{0} \rightarrow \mathbb{Z}\right)$.

Lemma 2. For every $i \geq 0$ the following groups are isomorphic:

$$
\left[K_{i}, K_{i}\right] \cong\left[\Omega^{i+1} \mathbb{Z}, \Omega^{i+1} \mathbb{Z}\right] .
$$

Proof: This follows from Shanuel's Lemma and an application of the fact that for arbitrary $M, N$ and projective modules $P$ and $Q$,

$$
[M \oplus P, N] \cong[M, N] \cong[M, N \oplus Q] .
$$

Proof of Proposition 1: Consider a partial projective resolution of $\mathbb{Z}$ over $\mathbb{Z} G$ where all $P_{i}, i \leq n-1$, are finitely generated:

$$
P_{n-1} \rightarrow \cdots \rightarrow P_{0} \rightarrow \mathbb{Z} \rightarrow 0,
$$

and let $K$ be the kernel of the map $P_{n-1} \rightarrow P_{n-2}$. As $G$ is of type $F P_{n}$ the kernel $K$ is finitely generated. Since tensoring with $\mathbb{Q}$ is exact we obtain a projective resolution of $\mathbb{Q}$ over $\mathbb{Q} G$, which is of type $F P$ :

$$
0 \rightarrow K \otimes \mathbb{Q} \rightarrow P_{n-1} \otimes \mathbb{Q} \rightarrow \cdots \rightarrow P_{0} \otimes \mathbb{Q} \rightarrow \mathbb{Q} \rightarrow 0 .
$$

Therefore $K \otimes \mathbb{Q}$ is a direct summand of a finite rank $\mathbb{Q} G$-free module $F$, freely generated by $\left\{f_{1}, \ldots, f_{r}\right\}$, say. Let $F_{0}$ be the free $\mathbb{Z} G$-module on these generators.

Claim. There is an integer $m$, such that multiplication with $m$ from $K$ to $K$ factors through $F_{0}$.

Let $\pi: F \rightarrow K \otimes \mathbb{Q}$ be the projection onto $K \otimes \mathbb{Q}$ and $\tau: K \otimes \mathbb{Q} \hookrightarrow F$ be a splitting, i.e., a map such that $\pi \tau=\operatorname{id}_{K \otimes \mathbb{Q}}$. Denote by $\iota: K \hookrightarrow K \otimes \mathbb{Q}$ the inclusion defined by $\iota(k)=k \otimes 1$. Suppose $k_{1}, \ldots, k_{s}$ generate $K$. For each $1 \leq j \leq s$ there exist $\lambda_{i j} \in \mathbb{Q} G$ such that $\iota \tau\left(k_{j}\right)=\sum_{i=1}^{r} \lambda_{i j} f_{i}$. Now pick $m \in \mathbb{Z}$ such that each $m \lambda_{i j} \in \mathbb{Z} G$. Since $\tau$ is a split injection we can precompose the identity $\operatorname{id}_{K \otimes \mathbb{Q}}=\pi \tau$ with multiplication by $m$. Hence the map

$$
K \stackrel{\iota}{\longrightarrow} K \otimes \mathbb{Q} \stackrel{\times m}{\longrightarrow} K \otimes \mathbb{Q}
$$

factors through $F_{0}$ and has image in $K$ thus proving the claim.

The claim together with Lemma 2 gives that

$$
m[K, K] \cong m\left[\Omega^{n} \mathbb{Z}, \Omega^{n} \mathbb{Z}\right]=0 .
$$


Complete cohomology agrees with ordinary Tate cohomology for finite groups and we can therefore take an arbitrary finite subgroup $H$ of $G$ and get that

$$
m \widehat{H}^{0}(H, \mathbb{Z}) \cong m[K, K] \cong \varliminf_{\longrightarrow} m\left[\Omega^{i} \mathbb{Z}, \Omega^{i} \mathbb{Z}\right]=0 .
$$

The direct limit vanishes since for every $\varphi \in \operatorname{Hom}\left(\Omega^{i} \mathbb{Z}, \Omega^{i} \mathbb{Z}\right)$, which factors through a projective, the induced maps $\Omega^{j} \varphi: \Omega^{i+j} \mathbb{Z} \rightarrow \Omega^{i+j} \mathbb{Z}$ also factor through projectives. (Note that $\Omega^{i} \mathbb{Z}$ here denotes the $i$ th kernel in the Benson-Carlson construction for $\mathbb{Z} H$ and not $\mathbb{Z} G$ as earlier used. This does not change the outcome, though.) But also $\widehat{H}^{0}(H, \mathbb{Z}) \cong \mathbb{Z} /|H| \mathbb{Z}$ and therefore the group order is a divisor of $m$, thus bounded.

\section{FP-groups over $\mathbb{Q}$}

Let us consider again the partial resolution of the $R$-module $M$ of type $F P_{n}$, which was mentioned at the beginning of the previous section:

$$
P_{n-1} \rightarrow P_{n-2} \rightarrow \cdots \rightarrow P_{1} \rightarrow P_{0} \rightarrow M \rightarrow 0 .
$$

There is such a partial resolution in which each $P_{i}$ is finitely generated and free. If also $M$ has projective dimension $n$, then $M$ is $F P$. If $M$ has projective dimension $n$ and the $P_{i}$ are finitely generated free modules, then $M$ is $F L$ if and only if $K$ is stably free. These results can be found in [3, Sections VIII.4-VIII.6]. The following lemma is well-known, but we could not find a reference, so we briefly sketch a proof. A similar topological result appears in [8, Corollary 5.5].

Lemma 3. Let $C$ denote an infinite cyclic group. For any $R$, if $G$ is a group of type FP over $R$, then $G \times C$ is of type $F L$ over $R$.

Proof: There is a free resolution $Q_{*}$ of $R$ over $R C$ of length one, with $Q_{1} \cong Q_{0} \cong R C$. Now suppose that

$$
0 \rightarrow P_{n} \rightarrow P_{n-1} \rightarrow \cdots \rightarrow P_{0} \rightarrow R \rightarrow 0
$$

is a projective resolution of $R$ over $R G$ in which each $P_{i}$ is finitely generated, and $P_{i}$ is free for $i<n$. Let $P^{\prime}$ be such that $P_{n} \oplus P^{\prime}$ is a finitely-generated free $R G$-module. Writing $\otimes$ for tensor products over $R$, the total complex $T_{*}$ for the double complex $P_{*} \otimes Q_{*}$ is a projective resolution of $R \otimes R=R$ over $R G \otimes R C \cong R(G \times C)$, of length $n+1$. Each $T_{i}$ is finitely generated and $T_{i}$ is free for $i<n$. Let $S_{*}$ be the exact chain complex consisting of one copy of $P^{\prime} \otimes R C$ in degree $n+1$ and one copy in degree $n$, with the identity map as the boundary. Then $S_{*} \oplus T_{*}$ is a finite free resolution of $R$ over $R(G \times C)$. 
Lemma 4. Let $F_{n} \rightarrow \cdots \rightarrow F_{0}$ be a finite-length chain complex of free $\mathbb{Z} G$-modules, suppose that $H_{0}\left(F_{*}\right)$ is isomorphic to the trivial $\mathbb{Z} G$-module $\mathbb{Z}$, and that for each $j>0$, there exists an integer $m_{j}>0$ such that multiplication by $m_{j}$ annihilates $H_{j}\left(F_{*}\right)$. Then any finite subgroup of $G$ has order dividing $\prod_{j=1}^{n} m_{j}$.

Sketch-proof: The above bound is obtained by comparing the two spectral sequences arising from the double complex

$$
E_{0}^{i, j}=\operatorname{Hom}_{H}\left(P_{i}, F_{j}\right)
$$

where $H$ is a finite subgroup of $G$ and $P_{*}$ is a complete resolution for $H$.

These lemmas can be used to prove a slightly weaker version of Proposition 1 using only ordinary Tate cohomology for finite groups. Suppose that $G$ is $F P_{n}$ over $\mathbb{Z}, F P$ over $\mathbb{Q}$, and $\operatorname{cd}_{\mathbb{Q}}(G)=n-1$. By Lemma 3 , $G^{\prime}=G \times C$ is $F P_{n}$ over $\mathbb{Z}, F L$ over $\mathbb{Q}$, and $\operatorname{cd}_{\mathbb{Q}}\left(G^{\prime}\right)=n$. A sequence of free $\mathbb{Z} G^{\prime}$-modules satisfying the conditions of Lemma 4 can then be constructed.

Let us now consider the problem of bounding the orders of finite subgroups of an arbitrary group of type $F P$ over $\mathbb{Q}$. Such a $G$ is finitely generated, and by Lemma 3, we may assume without loss of generality that $G$ is $F L$ over $\mathbb{Q}$. Let $P_{0}$ be a free $\mathbb{Q} G$-module of rank one with generator $v$, and let $P_{1}$ be $\mathbb{Q} G$-free on a set $e_{1}, \ldots, e_{m}$ bijective with a set $g_{1}, \ldots, g_{m}$ of generators for $G$. Define a map from $P_{0}$ to $\mathbb{Q}$ by $v \mapsto 1$ and a map from $P_{1}$ to $P_{0}$ by $e_{i} \mapsto\left(1-g_{i}\right) v$. Finally, let

$$
0 \rightarrow P_{n} \rightarrow \cdots \rightarrow P_{1} \rightarrow P_{0} \rightarrow \mathbb{Q} \rightarrow 0
$$

be a finite free resolution of $\mathbb{Q}$ over $\mathbb{Q} G$ extending this partial resolution.

Now let $F_{0}\left(\right.$ resp. $\left.F_{1}\right)$ be the $\mathbb{Z} G$-submodule of $P_{0}$ (resp. $\left.P_{1}\right)$ generated by $v$ (resp. $\left.e_{1}, \ldots, e_{m}\right)$. For $i \geq 2$, if $F_{i-1}$ has already been chosen, let $F_{i}$ be a $\mathbb{Z} G$-lattice in $P_{i}$ (i.e., a $\mathbb{Z} G$-free $\mathbb{Z} G$-submodule such that $\mathbb{Q} \otimes F_{i}=P_{i}$ ), such that the image of $F_{i}$ in $P_{i-1}$ is contained in $F_{i-1}$. This defines a finite chain complex $F_{*}$ of finitely-generated free $\mathbb{Z} G$-modules such that $H_{0}\left(F_{*}\right) \cong \mathbb{Z}$ and $H_{i}\left(F_{*}\right)$ is torsion for $i>0$. If one could bound the exponent of the torsion in $H_{i}\left(F_{*}\right)$, Lemma 4 could be applied to bound the orders of finite subgroups of $G$. Note that in general $H_{i}\left(F_{*}\right)$ will not be finitely generated as $\mathbb{Z} G$-module. For example, if $G$ is not $F P_{2}$ over $\mathbb{Z}$, then $H_{1}\left(F_{*}\right)$ will not be finitely generated.

Acknowledgement. The authors thank the referee for carefully checking an earlier version of this article. 


\section{References}

[1] D. J. Benson and J. F. CARlson, Products in negative cohomology, J. Pure Appl. Algebra 82(2) (1992), 107-129.

[2] R. BIERI, "Homological dimension of discrete groups", Queen Mary College Mathematics Notes, Mathematics Department, Queen Mary College, London, 1976.

[3] K. S. Brown, "Cohomology of groups", Graduate Texts in Mathematics 87, Springer-Verlag, New York, 1982.

[4] K. S. Brown, Finiteness properties of groups, in: "Proceedings of the Northwestern conference on cohomology of groups" (Evanston, Ill., 1985), J. Pure Appl. Algebra 44 (1987), 45-75.

[5] C. H. Houghton, The first cohomology of a group with permutation module coefficients, Arch. Math. (Basel) 31(3) (1978/79), 254-258.

[6] P. H. Kropholler, On groups of type $(\mathrm{FP})_{\infty}$, J. Pure Appl. Algebra 90(1) (1993), 55-67.

[7] I. J. Leary And B. E. A. Nucinkis, Some groups of type VF, Preprint (2000).

[8] G. MisLin, Wall's finiteness obstruction, in "Handbook of algebraic topology", North-Holland, Amsterdam, 1995, pp. 1259-1291.

[9] G. Mislin, Tate cohomology for arbitrary groups via satellites, Topology Appl. 56(3) (1994), 293-300.

Faculty of Mathematical Studies

University of Southampton

Southampton SO17 1BJ

United Kingdom

E-mail address: I.J.Leary@maths.soton.ac.uk

Department of Mathematics

ETH-Zentrum

8092 Zürich

Switzerland

E-mail address: bean@math.ethz.ch

Primera versió rebuda el 18 de desembre de 2000, darrera versió rebuda el 23 de gener de 2001. 